\title{
The genomic architecture of NLRP7 is Alu rich and predisposes to disease-associated large deletions
}

\author{
Ramesh Reddy, Ngoc MP Nguyen, Guillaume Sarrabay, Maryam Rezaei, Mayra CG Rivas, \\ Aysenur Kavasoglu, Hakan Berkil, Alaa Elshafey, Ebtesam Abdalla, Kristin P Nunez, Hélène Dreyfus, \\ Merviel Philippe, Zahra Hadipour, Asude Durmaz, Erin E Eaton, Brittany Schubert, Volkan Ulker, \\ Fatemeh Hadipour, Isabelle Touitou, Majid Fardaei and Rima Slim
}

European Journal of Human Genetics (2016) 24, 1516; doi:10.1038/ejhg.2016.96

Correction to: European Journal of Human Genetics (2016) 24, 1445-1452; doi:10.1038/ejhg.2016.9; published online 9 March 2016
The name of Dr Ebtesam Abdalla was inadvertently omitted from the Author list. This has now been added to the article. The affiliations have also been amended accordingly.

The authors would like to apologise for their error. 